Article

\title{
General Approach for Composite Thermoelectric Systems with Thermal Coupling: The Case of a Dual Thermoelectric Cooler
}

\author{
Cuautli Yanehowi Flores-Niño, Miguel Angel Olivares-Robles * and Igor Loboda \\ Instituto Politecnico Nacional, SEPI ESIME-CUL, Av. Santa Ana 1000, Culhuacan, Coyoacan 04430, \\ Mexico; E-Mail: iloboda@ipn.mx (I.L.) \\ * Author to whom correspondence should be addressed; E-Mail: olivares@ipn.mx; \\ Tel.: +52-555-729-6000 (ext 73262); Fax: +52-555-656-2058.
}

Academic Editor: Kevin H. Knuth

Received: 17 April 2015 / Accepted: 3 June 2015 / Published: 8 June 2015

\begin{abstract}
In this work, we show a general approach for inhomogeneous composite thermoelectric systems, and as an illustrative case, we consider a dual thermoelectric cooler. This composite cooler consists of two thermoelectric modules (TEMs) connected thermally in parallel and electrically in series. Each TEM has different thermoelectric (TE) properties, namely thermal conductance, electrical resistance and the Seebeck coefficient. The system is coupled by thermal conductances to heat reservoirs. The proposed approach consists of derivation of the dimensionless thermoelectric properties for the whole system. Thus, we obtain an equivalent figure of merit whose impact and meaning is discussed. We make use of dimensionless equations to study the impact of the thermal conductance matching on the cooling capacity and the coefficient of the performance of the system. The equivalent thermoelectric properties derived with our formalism include the external conductances and all intrinsic thermoelectric properties of each component of the system. Our proposed approach permits us changing the thermoelectric parameters of the TEMs and the working conditions of the composite system. Furthermore, our analysis shows the effect of the number of thermocouples on the system. These considerations are very useful for the design of thermoelectric composite systems. We reproduce the qualitative behavior of a commercial composite TEM connected electrically in series.
\end{abstract}

Keywords: thermoelectric cooler, Peltier effect; cooling capacity; coefficient of performance; thermal coupling 


\section{Introduction}

Composite thermoelectric systems, based on thermoelectric effects, such as Seebeck effect and Peltier effect, have a variety of uses nowadays. A composite thermoelectric cooling system (composite TECS) has many advantages in comparison with the traditional cooling systems, such as the lack of moving parts, low weight, does not need maintenance and the fact that it is environmentally friendly due to lack of cooling substances. Disadvantage of the TECS are the low cooling capacity $Q_{c}$ and coefficient of performance (COP). New techniques and thermoelectric materials have been proposed for designing new thermoelectric specific systems that allow the improvement of the device performance through the optimization of the thermal and electrical transport properties [1-3]. These TECS, known as solid-state devices, are used in many different applications, ranging from controlling the temperature of laser diodes, infrared detectors, superconductor applications, aerospace applications, electronic devices and food storage [4].

It is well known that the thermal conductance of ceramic plates plays a vital role in the performance of TECS. Yamanashi [5] has considered the effects of the thermal resistance of heat exchangers on the performance of a TEC, with a constant thermocouple number. He has obtained the design parameters through the dimensionless heat balance equations. On the other hand, Xuan [6], through introducing equivalent impedances to take into account the thermal contact effect of a single stage, have derived the maximum temperature difference, cooling capacity and COP. Recently, Pearson and Lents [7] have studied a thermal network with an integrated TEC and performed a dimensionless analysis. They concluded that dimensionless parameters reduce the complexity of the results, enabling the evaluation of the system without knowing the detailed information of the geometries or the materials. These proposals consider homogeneous TECS, i.e., TECS with thermoelectric modules (TEMs) that have the same thermoelectric properties, namely the Seebeck coefficient, electrical resistance and thermal conductance. However, the behavior of inhomogeneous composite TECS, i.e., composite TECS with TEMs that have different thermoelectric properties, can not be predicted.

Apertet et al. [8] have considered an inhomogeneous thermoelectric generator system (TEGS) composed of two different thermoelectric modules, electrically and thermally connected in parallel, using linear irreversible thermodynamics, and they have proposed equivalent parameters for an equivalent thermoelectric generator system, including realistic thermal coupling [9]. Using this linear approach, others authors $[10,11]$ have considered different configurations for composite TEGS.

Moreover, a widely-considered approach in the analysis for both TECS and TEGS is Ioffe's approach [12]. This approach includes the Joulean heat loss [5,6]. Thus, we are interested in the analysis of inhomogeneous composite TECS, and we propose a general approach, based in Ioffe's approach, which includes all thermoelectric properties of the TEMs constituting the TECS. We illustrate this general approach for a dual thermoelectric cooling system that consists of two TEMs with different thermoelectric properties and thermal coupling. This approach can be useful for designing a composite solid-state device with thermal coupling for switching between low power consumption or high heat pumping performance [13].

The paper is organized as follows: In Section 2, we present the configuration of the dual thermoelectric cooler system. We derive the equivalent parameters for our system and transform it into 
its dimensionless form, in Section 3. In Section 4, we analyze our numerical results for $C O P$ and for cooling capacity, $Q_{C}$, and their behavior as a function of the thermal impedances. Finally, in Section 5, we present our conclusions, and future work is discussed briefly.

\section{Dual Thermoelectric Cooling System}

Our composite TEC system is composed of two thermoelectric modules thermally connected in parallel and electrically connected in series, as depicted in Figure 1. The system is coupled to two heat reservoirs by thermal exchangers, and the temperatures of the heat reservoirs are $T_{c}$ and $T_{h}$ with $T_{h}>T_{c} . K_{c}$ and $K_{h}$ are the thermal conductances of the heat exchangers at both sides, cold and hot, respectively. Each TEM is characterized by a Seebeck coefficient $\alpha_{i}$, thermal conductance $K_{i}$ and an electrical resistance $R_{i}$, where $i$ can be one or two as appropriate. The TEM 1 and TEM 2 are formed by $m$ and $n$ thermocouples number, respectively. $I$ is the electrical current through the TEMs.

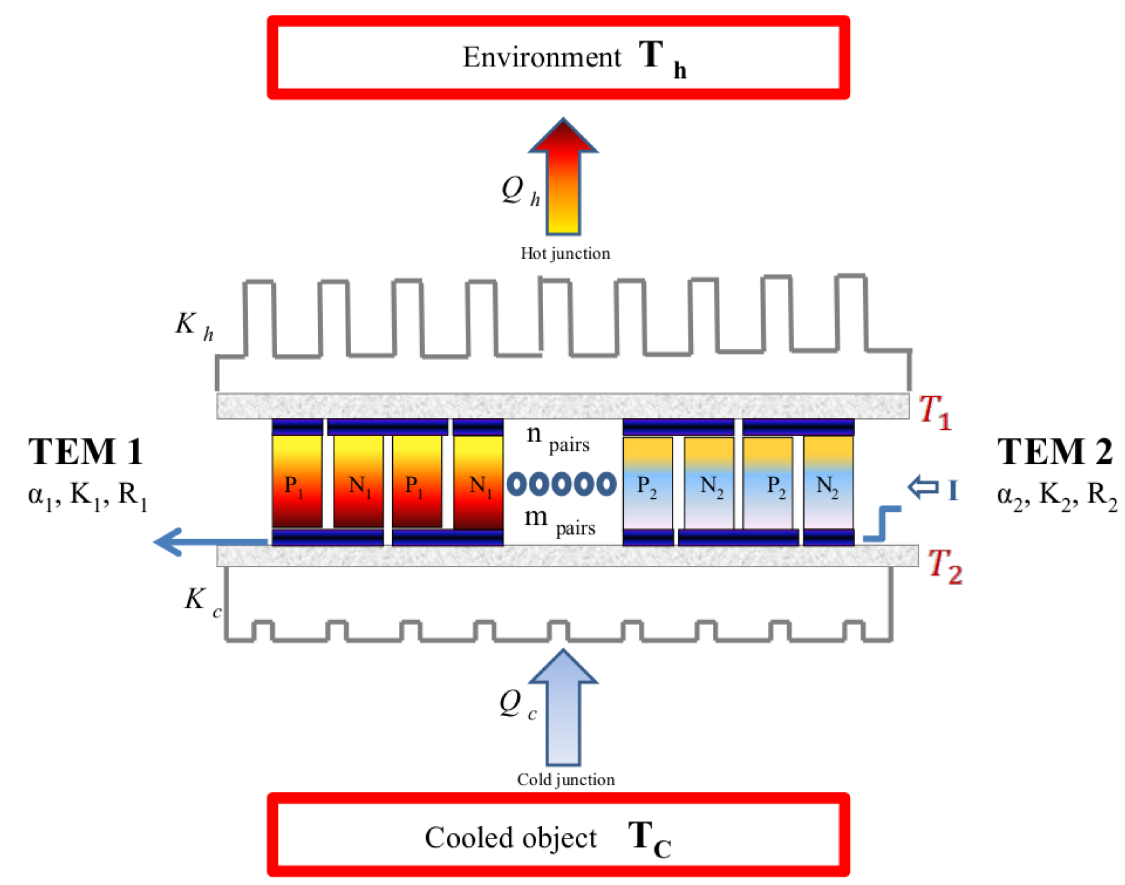

Figure 1. Schematic of a Dual Thermoelectric Cooling System.

\section{Heat Balance Equations}

We assume that the heat flow between the TEMs and the surroundings is ignored, except for the cold and hot end of each TEM; also, the properties of the $N$ - and $P$-type elements are assumed independent of temperature. In Figure $1, Q_{h}$ is the heat rejected from the TEC system to the heat reservoir and $Q_{c}$ is the cooling capacity or the heat absorbed from the cooled object. Assuming that the heat flow between each stage and the heat reservoirs obey Newton's law, we have:

$$
\begin{aligned}
Q_{h} & =K_{h}\left(T_{1}-T_{h}\right) \\
Q_{c} & =K_{c}\left(T_{c}-T_{2}\right)
\end{aligned}
$$


The heat flux flowing through the dual TEC system is the sum of two heat fluxes flowing through $\mathrm{TEM}_{1}$ and $\mathrm{TEM}_{2}$,

$$
\begin{aligned}
& Q_{h}=Q_{h 1}+Q_{h 2} \\
& Q_{c}=Q_{c 1}+Q_{c 2}
\end{aligned}
$$

The heat fluxes, $Q_{c i}$ and $Q_{h i}(i=1,2)$, for each TEM are given by:

$$
\begin{aligned}
Q_{h 1} & =m\left[\alpha_{1} I T_{1}+\frac{1}{2} I^{2} R_{1}-K_{1}\left(T_{1}-T_{2}\right)\right] \\
Q_{c 1} & =m\left[\alpha_{1} I T_{2}-\frac{1}{2} I^{2} R_{1}-K_{1}\left(T_{1}-T_{2}\right)\right] \\
Q_{h 2} & =n\left[\alpha_{2} I T_{1}+\frac{1}{2} I^{2} R_{2}-K_{2}\left(T_{1}-T_{2}\right)\right] \\
Q_{c 2} & =n\left[\alpha_{2} I T_{2}-\frac{1}{2} I^{2} R_{2}-K_{2}\left(T_{1}-T_{2}\right)\right]
\end{aligned}
$$

where $m$ and $n$ are the number of thermocouples for $\mathrm{TEM}_{1}$ and $\mathrm{TEM}_{2}$, respectively. Equations (5)-(8) contain three terms, namely $\alpha I T$, Peltier heat, $I^{2} R$, the internal heat generated by the Joulean loss and $K\left(T_{1}-T_{2}\right)$, conduction heat loss. Clearly, the properties of different thermoelectric semiconductor materials of each TEM are included in the above equations.

\subsection{Equivalent Dual TEC System}

In this section, we derive the equivalent thermoelectric parameters of the dual TEC system as a whole. Combining Equations (1)-(3), (5) and (7), we obtain for the heat rejected, $Q_{h}$,

$$
Q_{h}=\alpha_{e q H} I T_{h}+\frac{1}{2} I^{2} R_{e q H}-K_{e q H}\left(T_{h}-T_{c}\right)
$$

where,

$$
\begin{aligned}
\alpha_{e q H} & =\frac{\left(m \alpha_{1}+n \alpha_{2}\right)\left(1+\frac{m \alpha_{1} I+n \alpha_{2} I}{K_{c}}\right)}{1+\frac{\left(m K_{1}+n K_{2}\right)\left(K_{c}+K_{h}\right)}{K_{h} K_{c}}+\frac{\left(m \alpha_{1} I+n \alpha_{2} I\right)\left(K_{h}-K_{c}\right)}{K_{h} K_{c}}-\frac{\left(m \alpha_{1} I+n \alpha_{2} I\right)^{2}}{K_{h} K_{c}}} \\
R_{e q H} & =\frac{\left(m R_{1}+n R_{2}\right)\left(1+\frac{m \alpha_{1} I+n \alpha_{2} I}{K_{c}}+2 \frac{m K_{1}+n K_{2}}{K_{c}}\right)}{1+\frac{\left(m K_{1}+n K_{2}\right)\left(K_{c}+K_{h}\right)}{K_{h} K_{c}}+\frac{\left(m \alpha_{1} I+n \alpha_{2} I\right)\left(K_{h}-K_{c}\right)}{K_{h} K_{c}}-\frac{\left(m \alpha_{1} I+n \alpha_{2} I\right)^{2}}{K_{h} K_{c}}} \\
K_{e q H} & =\frac{\left(m K_{1}+n K_{2}\right)}{1+\frac{\left(m K_{1}+n K_{2}\right)\left(K_{c}+K_{h}\right)}{K_{h} K_{c}}+\frac{\left(m \alpha_{1} I+n \alpha_{2} I\right)\left(K_{h}-K_{c}\right)}{K_{h} K_{c}}-\frac{\left(m \alpha_{1} I+n \alpha_{2} I\right)^{2}}{K_{h} K_{c}}}
\end{aligned}
$$

Similarly, combining Equations (1), (2), (4), (6) and (8), we have for absorbed heat $Q_{c}$,

$$
Q_{c}=\alpha_{e q C} I T_{c}-\frac{1}{2} I^{2} R_{e q C}-K_{e q C}\left(T_{h}-T_{c}\right)
$$

where:

$$
\begin{aligned}
\alpha_{e q C} & =\frac{\left(m \alpha_{1}+n \alpha_{2}\right)\left(1-\frac{m \alpha_{1} I+n \alpha_{2} I}{K_{h}}\right)}{1+\frac{\left(m K_{1}+n K_{2}\right)\left(K_{c}+K_{h}\right)}{K_{h} K_{c}}+\frac{\left(m \alpha_{1} I+n \alpha_{2} I\right)\left(K_{h}-K_{c}\right)}{K_{h} K_{c}}-\frac{\left(m \alpha_{1} I+n \alpha_{2} I\right)^{2}}{K_{h} K_{c}}} \\
R_{e q C} & =\frac{\left(m R_{1}+n R_{2}\right)\left(1-\frac{m \alpha_{1} I+n \alpha_{2} I}{K_{h}}+2 \frac{m K_{1}+n K_{2}}{K_{h}}\right)}{1+\frac{\left(m K_{1}+n K_{2}\right)\left(K_{c}+K_{h}\right)}{K_{h} K_{c}}+\frac{\left(m \alpha_{1} I+n \alpha_{2} I\right)\left(K_{h}-K_{c}\right)}{K_{h} K_{c}}-\frac{\left(m \alpha_{1} I+n \alpha_{2} I\right)^{2}}{K_{h} K_{c}}} \\
K_{e q C} & =\frac{\left(m K_{1}+n K_{2}\right)}{1+\frac{\left(m K_{1}+n K_{2}\right)\left(K_{c}+K_{h}\right)}{K_{h} K_{c}}+\frac{\left(m \alpha_{1} I+n \alpha_{2} I\right)\left(K_{h}-K_{c}\right)}{K_{h} K_{c}}-\frac{\left(m \alpha_{1} I+n \alpha_{2} I\right)^{2}}{K_{h} K_{c}}}
\end{aligned}
$$


The equivalent thermoelectric parameters of our dual TEC system are given by Equations (10)-(12) and (14)-(16). These equations generalize the previously obtained results for homogeneous thermoelectric cooling systems considered by other authors [6]. For example, the parameters $\alpha_{e q H}$ and $\alpha_{e q C}$ are the effective Seebeck coefficients for the hot and cold side, respectively. These equivalent parameters not only combine all intrinsic thermoelectric properties of both modules, but also they are significantly influenced by external thermal conductances. In Section 4.4, we will show that the numerical behavior of the figure of merit for the whole system satisfies Bergman's theorem. Notice that the equivalent heat balance equations of the system (9) and (13), depend only on the temperatures $T_{c}$ and $T_{h}$. Furthermore, we will use these results and recover, as limit cases, the design parameters for TEC system, which have been previously studied by other authors; see Section 4.5.

\subsection{Dimensionless Equivalent Heat Balance Equations}

When a TEC system is optimized, it is convenient to rewrite the heat balance Equations (9) and (13), into the dimensionless form as follows [5],

$$
\begin{aligned}
& q_{h}=\varphi \frac{1+\varphi \delta}{\mu}+\frac{1}{2} \varphi^{2} \frac{1}{Z_{e q} T_{h}} \frac{1+\varphi \delta+2 \delta}{\mu}-\frac{1-\theta}{\mu} \\
& q_{c}=\varphi \frac{1-\varphi \rho}{\mu}-\frac{1}{2} \varphi^{2} \frac{1}{Z_{e q} T_{c}} \frac{1-\varphi \rho+2 \rho}{\mu}-\frac{\frac{1}{\theta}-1}{\mu}
\end{aligned}
$$

where dimensionless quantities are defined as,

$$
\begin{aligned}
\theta & =\frac{T_{c}}{T_{h}} \\
\delta & =\frac{\left(m K_{1}+n K_{2}\right)}{K_{c}} \\
\rho & =\frac{\left(m K_{1}+n K_{2}\right)}{K_{h}} \\
\varphi & =I \frac{\left(m \alpha_{1}+n \alpha_{2}\right)}{\left(m K_{1}+n K_{2}\right)} \\
Z_{e q} & =\frac{\left(m \alpha_{1}+n \alpha_{2}\right)^{2}}{\left(m R_{1}+n R_{2}\right)\left(m K_{1}+n K_{2}\right)} \\
q_{h} & =\frac{Q_{h}}{\left(m K_{1}+n K_{2}\right) T_{h}} \\
q_{c} & =\frac{Q_{c}}{\left(m K_{1}+n K_{2}\right) T_{c}} \\
\mu & =1+\delta+\rho+(\delta-\rho) \varphi-\varphi^{2} \delta \rho
\end{aligned}
$$

In equations (17) and (18), the dimensionless current is represented by $\varphi$. The parameters $\delta$ and $\rho$ are dimensionless thermal conductances normalized by the cold and hot external thermal conductances, respectively. $\theta$ is the temperature ratio of heat reservoirs. $Z_{e q}$ is the equivalent figure of merit and $\mu$ is a coupling factor. Notice that we recover the dimensionless quantities $q_{h}$ and $q_{c}$, which have been considered as the entropy flow normalized by the intrinsic thermal conductances, $m K_{1}+n K_{2}$, of the inhomogeneous TEC system. 
The equivalent figure of merit $Z_{e q}$, Equation (23), can be written in terms of the number of pairs of each TEM as:

$$
Z_{e q}=\frac{\alpha_{1}^{2}\left(D+\sqrt{Z_{r}}\right)^{2}}{R_{1} K_{1}\left(D+Z_{r}\right)^{2}}=Z_{1} \frac{\left(D+\sqrt{Z_{r}}\right)^{2}}{\left(D+Z_{r}\right)^{2}}
$$

where the number of pairs ratio, $D$, and the figure of merit ratio, $Z_{r}$, are given by:

$$
\begin{gathered}
D=\frac{m}{n} \\
Z_{r}=\frac{Z_{1}}{Z_{2}}
\end{gathered}
$$

Now, the well-known COP for a cooling system,

$$
C O P=\frac{Q_{c}}{Q_{h}-Q_{c}}
$$

can be written in terms of dimensionless heat quantities,

$$
C O P=\frac{q_{c}}{\frac{1}{\theta} q_{h}-q_{c}}
$$

We point out that this approach can be used in the analysis of a thermoelectric heat pump.

\section{Results and Discussion}

In this section, we study the effect of the thermal conductance matching on the cooling capacity $q_{c}$ and the COP of the TEC system. In our calculations, we use values $T_{c}=286 \mathrm{~K}$ and $T_{h}=296 \mathrm{~K}$ for the temperatures of the cold and hot reservoirs, respectively.

\subsection{Cooling Capacity $q_{c}$ : External Conductances Match}

The behavior of the cooling capacity and the COP as a function of the dimensionless current, $\varphi$, for different external conductance ratios,

$$
K_{c h}=\frac{K_{c}}{K_{h}}
$$

are shown by Figures 2 and 6, respectively (see also Figures 11, 13 in Appendix).

Figure 2 shows that a limit value of $q_{c}$ is reached as the ratio $K_{c h}$ decreases for a given value of $\varphi$ when $K_{c}<K_{h}$. As the external conductance ratio decreases, the change in maximal values of the cooling capacity are determined by the electrical current $\varphi$. Moreover, the limit values of $q_{c}$ are determined by the ratio $K_{c h}$. Thus, the condition $K_{c}<K_{h}$ is bounded, because $q_{c}$ will reach limit values. Notice that it is possible to reach the same limit value of $q_{c}$, using different values of electrical current, $\varphi$.

From the results obtained in Section 3, our approach permits us to calculate the cooling capacity, $q_{c}$, in terms of any two thermoelectric parameters $\left(\varphi, K_{c h}, Z_{r}\right)$ of the equivalent TEC system. For example, Figure 3 shows the behavior of $q_{c}$ as a function of the figure of merit ratio $Z_{r}$ and external conductances ratio, $K_{c h}$, for different values of electrical current, $\varphi$. 


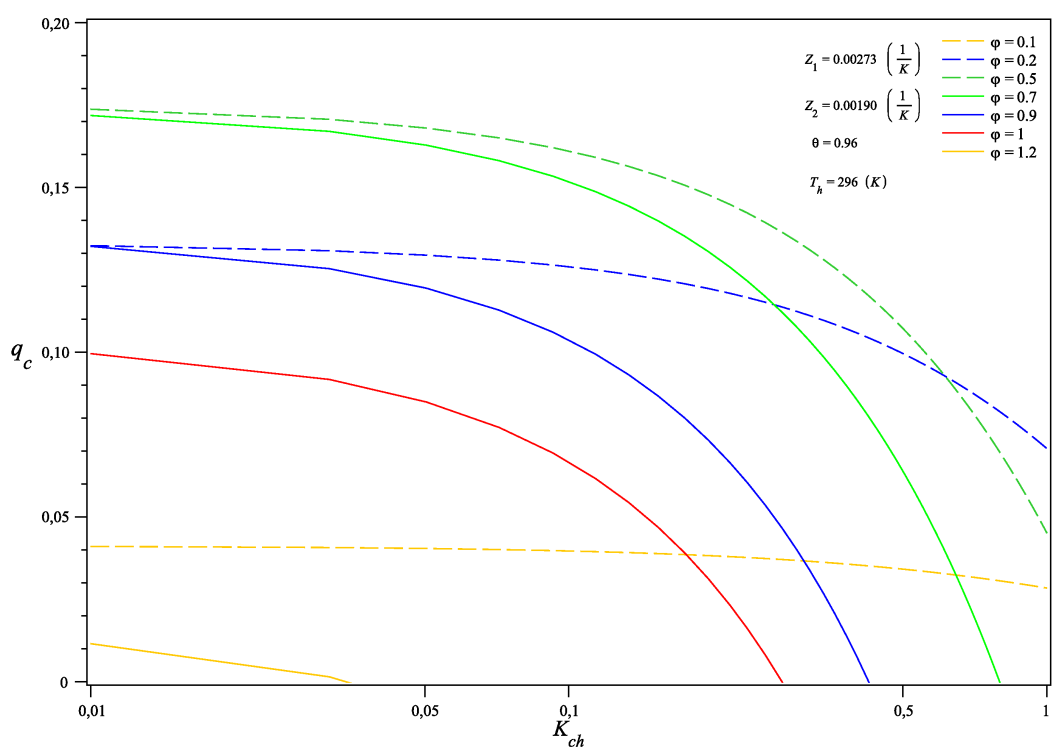

Figure 2. Cooling capacity $v s$. the external conductance ratio with different dimensionless currents.

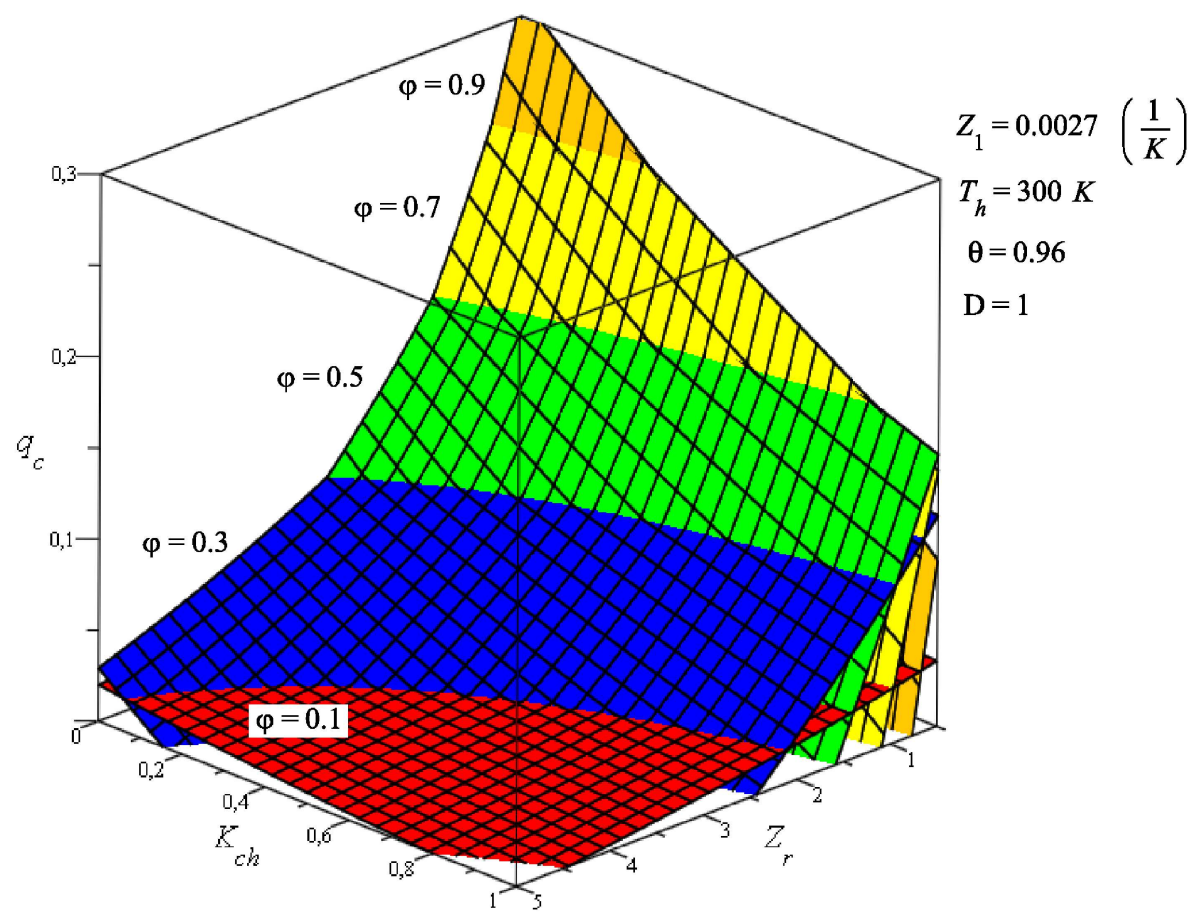

Figure 3. Cooling capacity vs. the external conductance ratio with different figure of merit ratios and dimensionless current.

Notice that the behavior of $q_{c}$ includes several effects that are contained in different planes of Figure 3. For example, maximum values for cooling capacity $q_{c}$ are shown in the plane $q_{c} v s . Z_{r}$, at different values of electrical current $\varphi$, for small figure of merit ratios, $Z_{r}$, and thermal conductances ratios, $K_{c h}$. This result is more clearly shown in Figure 4. 


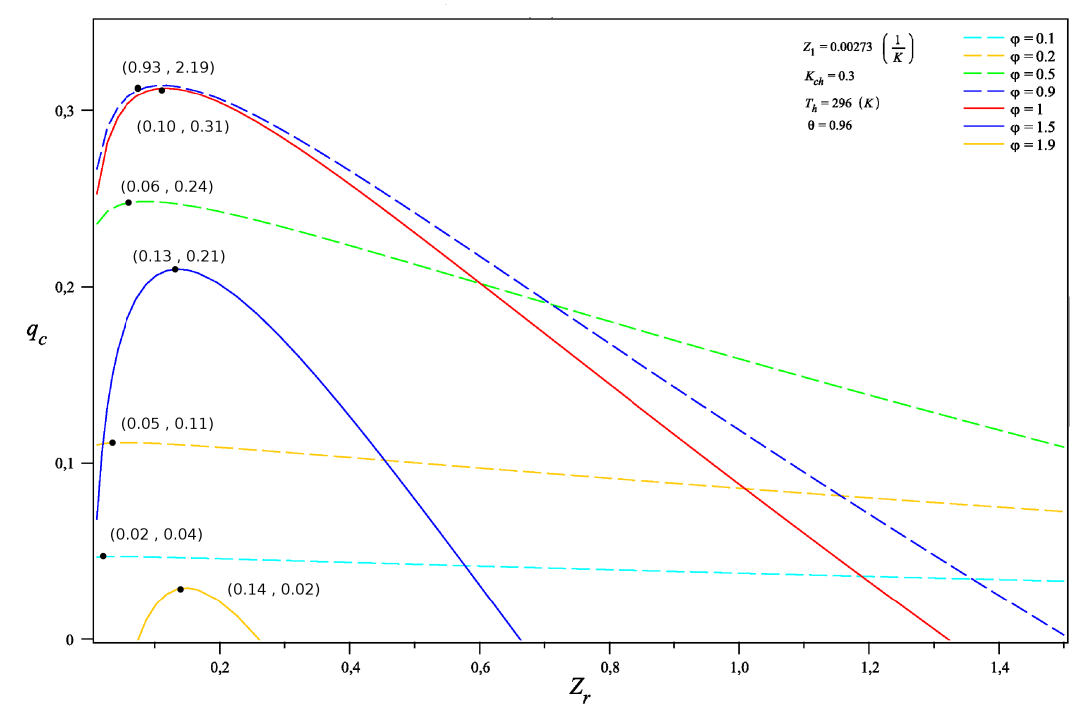

Figure 4. Cooling capacity $v s$. the figure of merit ratio with different dimensionless current.

Our approach permits us to know the effect of (1) thermal conductances, $K_{c h}$, and (2) the figure of merit ratio, $Z_{r}$, on the cooling capacity $q_{c}$ at different working conditions. These effects are shown in Figures 11 and 12 of the Appendix.

Furthermore, we show the effect on $q_{c}$ of both $\varphi$ and $Z_{r}$, in Figure 5, for a given value of $K_{c h}$.

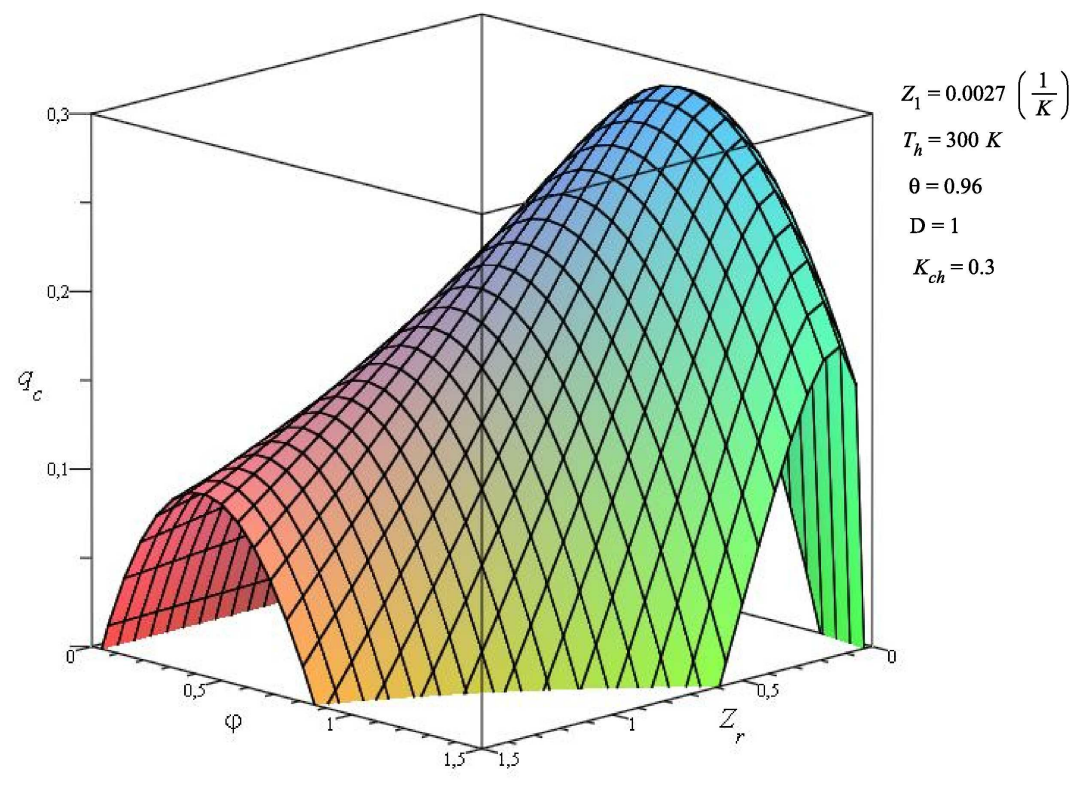

Figure 5. Cooling capacity $v s$. the figure of merit ratio and dimensionless current.

In fact, Figure 5 shows the optimal values of $q_{c}$ in terms of $\varphi$ and $Z_{r}$ for constant values of other parameters, namely $Z_{1}, T_{h}, \theta, K_{c h}$ and $D$, which determine the working conditions of the composite TEC system.

We point out that our proposed approach permits us to change the thermoelectric parameters of the TEMs and the working conditions of the composite system. This fact is very useful for the design of thermoelectric composite systems. 


\subsection{Coefficient of Performance (COP): External Conductances Match}

Analogously, Figures 6, 7 and 8 show the corresponding behavior for $C O P$ of the system as a function of $K_{c h}, Z_{r}$ and $\varphi$, respectively.

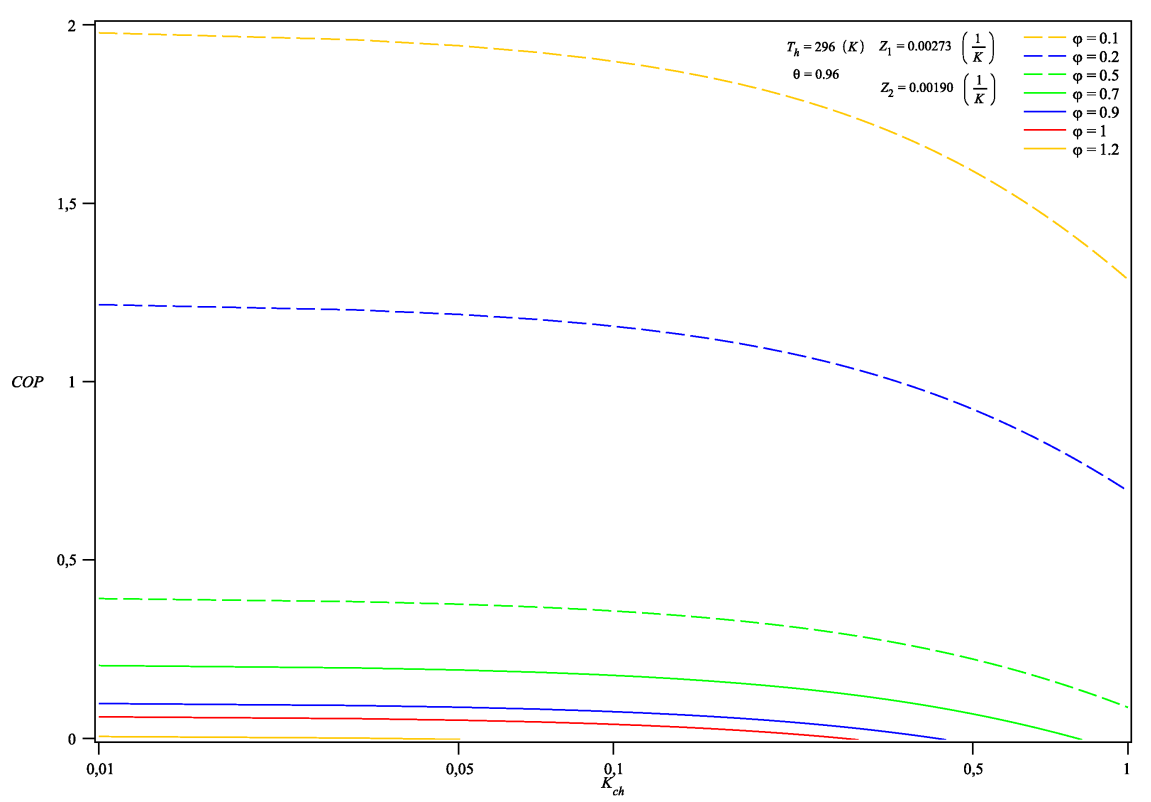

Figure 6. COP vs. the external conductance ratio with different dimensionless currents.

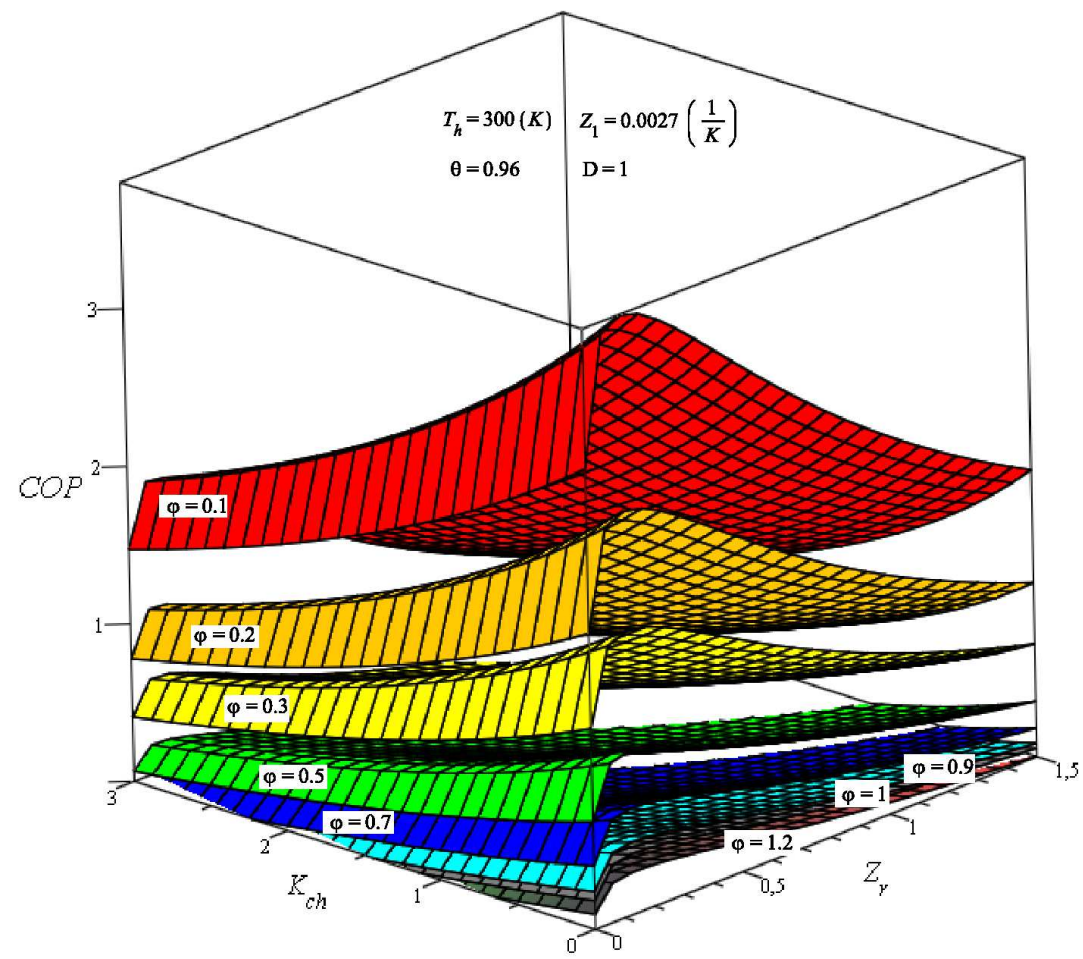

Figure 7. COP vs. the external conductances ratio with different figures of merit and dimensionless current. 


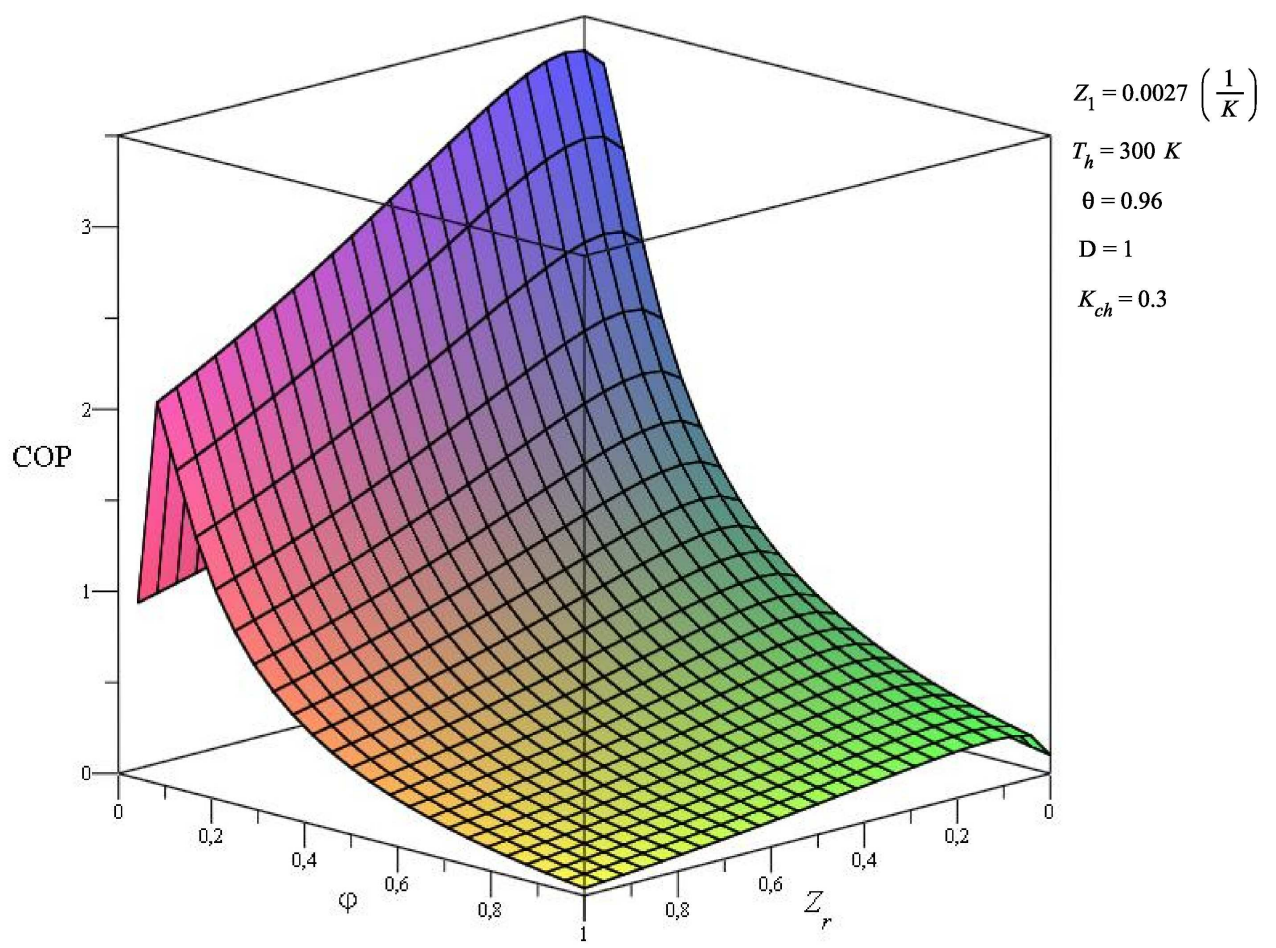

Figure 8. COP $v s$. the figure of merit ratio and dimensionless current.

Again, we obtain several results for $C O P$ of the system, which are included in different planes of Figures 7 and 8. See Figures 13, 14 and 15 in the Appendix.

Table 1. COP for different values $K_{c h}$.

\begin{tabular}{|c|c|c|}
\hline$K_{\text {ch }}$ & $C O P$ & $\Delta C O P$ \\
\hline 0 & 1.9866 & 0.045 \\
\hline 0.05 & 1.9416 & 0.0437 \\
\hline 0.10 & 1.8979 & 0.0424 \\
\hline 0.15 & 1.8555 & 0.0412 \\
\hline 0.20 & 1.8143 & 0.0400 \\
\hline 0.25 & 1.7743 & 0.0389 \\
\hline 0.30 & 1.7354 & 0.0378 \\
\hline 0.35 & 1.6976 & 0.0368 \\
\hline 0.40 & 1.6608 & 0.0358 \\
\hline 0.45 & 1.6250 & 0.0349 \\
\hline 0.50 & 1.5901 & 0.0339 \\
\hline 0.55 & 1.5562 & 0.0331 \\
\hline 0.60 & 1.5231 & 0.0322 \\
\hline 0.65 & 1.4909 & 0.0314 \\
\hline 0.70 & 1.4595 & 0.0306 \\
\hline 0.75 & 1.4289 & 0.0298 \\
\hline 0.80 & 1.3991 & 0.0292 \\
\hline 0.85 & 1.3699 & 0.0284 \\
\hline 0.90 & 1.3415 & 0.0278 \\
\hline 0.95 & 1.3137 & 0.0271 \\
\hline
\end{tabular}

For example, Figures 6 and 13 show that the maximal values of the $C O P$ tend to a limit maximum value as the ratio $K_{c h}$ changes. This fact shows that it is not possible to optimize the system by just 
fixing the $K_{c h}$, but also a limit for the external conductances $K_{c h}$ exists. Table 1 shows the behavior of limit values for $C O P$ as we change the ratio $K_{c h}$.

As is well known, the optimal working conditions are different for both maximum values of $q_{c}$ and $C O P$. A maximum value for $q_{c}$ is obtained for high values of $\varphi$; meanwhile, the maximum values of $C O P$ are obtained in low ranges of $\varphi$. In Section 4.3, we obtain the qualitative behavior of $C O P$ for a commercial composite TEM connected in series.

\subsection{Numerical Validation}

Firstly, Table 2 shows some values of $K_{c h}, \delta$ and $\rho$ used in our calculations, only for completeness reasons.

Table 2. Values of $\rho$ and $\delta$ for given values of $K_{c h}$.

\begin{tabular}{ccccc}
\hline$K_{\text {ch }}$ & 0.3 & 0.5 & 1 & 2 \\
\hline$\delta$ & 0.3522 & 0.3522 & 0.3522 & 0.3522 \\
$\rho$ & 0.10566 & 0.1761 & 0.3522 & 0.7044 \\
\hline
\end{tabular}

Nowadays, it is possible to fabricate composite TEMs connected in different configurations $[13,14]$. We use the thermoelectric parameters, $\alpha_{1}=\alpha_{2}=\alpha_{\text {Luo }}, R_{1}=R_{2}=R_{\text {Luo }}$ and $K_{1}=K_{2}=K_{\text {Luo }}$, for a composite TEM connected in series proposed by Luo [15] for obtaining Figure 9. The behavior of the $C O P$, shown in Figure 9, is very near to that reported in the datasheet for the $S P-254-$ $1.0-2.5$ (series) TEM connected electrically in series, with the same thermoelectric properties for each component of the TEM [16].

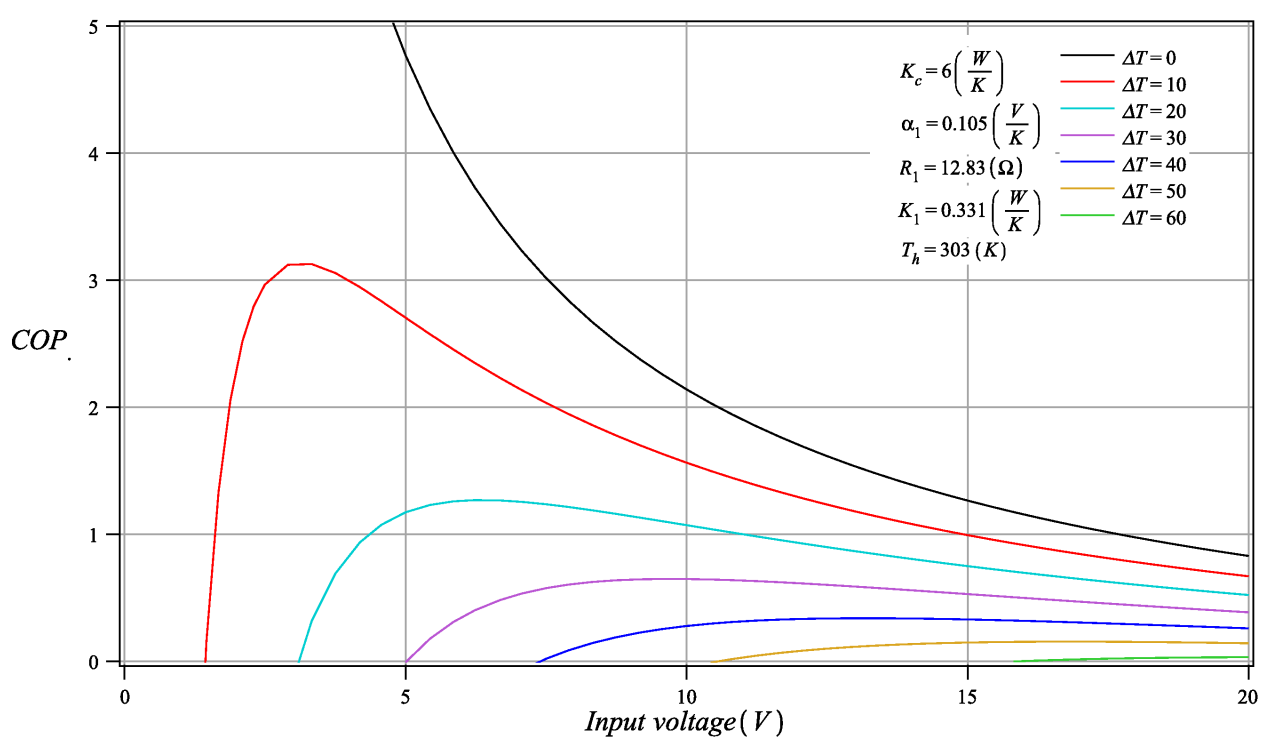

Figure 9. COP vs. the input voltage corresponding to an $S P-254-1.0-2.5$ (series) TEC module connected in series. 


\subsection{Role of the Equivalent Figure of Merit}

In the previous section, we studied the effect of the external conductance matching on the performance and the cooling capacity of our system. In this section, we analyze the role of the equivalent figure of merit, $Z_{e q}$, in the $q_{c}$ and COP parameters in terms of the number of pairs, $n$ and $m$, and the figure of merit of each component TEM, $Z_{1}$ and $Z_{2}$.

Using Equation (23) or Equation (27) and the numerical values of the thermoelectric properties of TEM 2, shown in Table 3, for a fixed value of $Z_{1}=0.0027$, we obtain Figure 10, which shows the equivalent figure of merit of the system as a function of the number pairs of each TEM.

Table 3. Numerical values of the thermoelectric properties of TEM 2 when $Z_{1}=0.0027$.

\begin{tabular}{ccccc}
\hline$Z_{r}$ & $\alpha_{2}$ & $R_{2}$ & $K_{2}$ & $Z_{2}$ \\
\hline 0.1 & 0.0170 & 0.341 & 0.0313 & 0.0273 \\
0.5 & 0.0381 & 1.705 & 0.1565 & 0.0054 \\
1 & 0.054 & 3.41 & 0.313 & 0.0027 \\
2 & 0.0766 & 6.82 & 0.626 & 0.0013 \\
3 & 0.0935 & 10.23 & 0.939 & 0.0009 \\
\hline
\end{tabular}

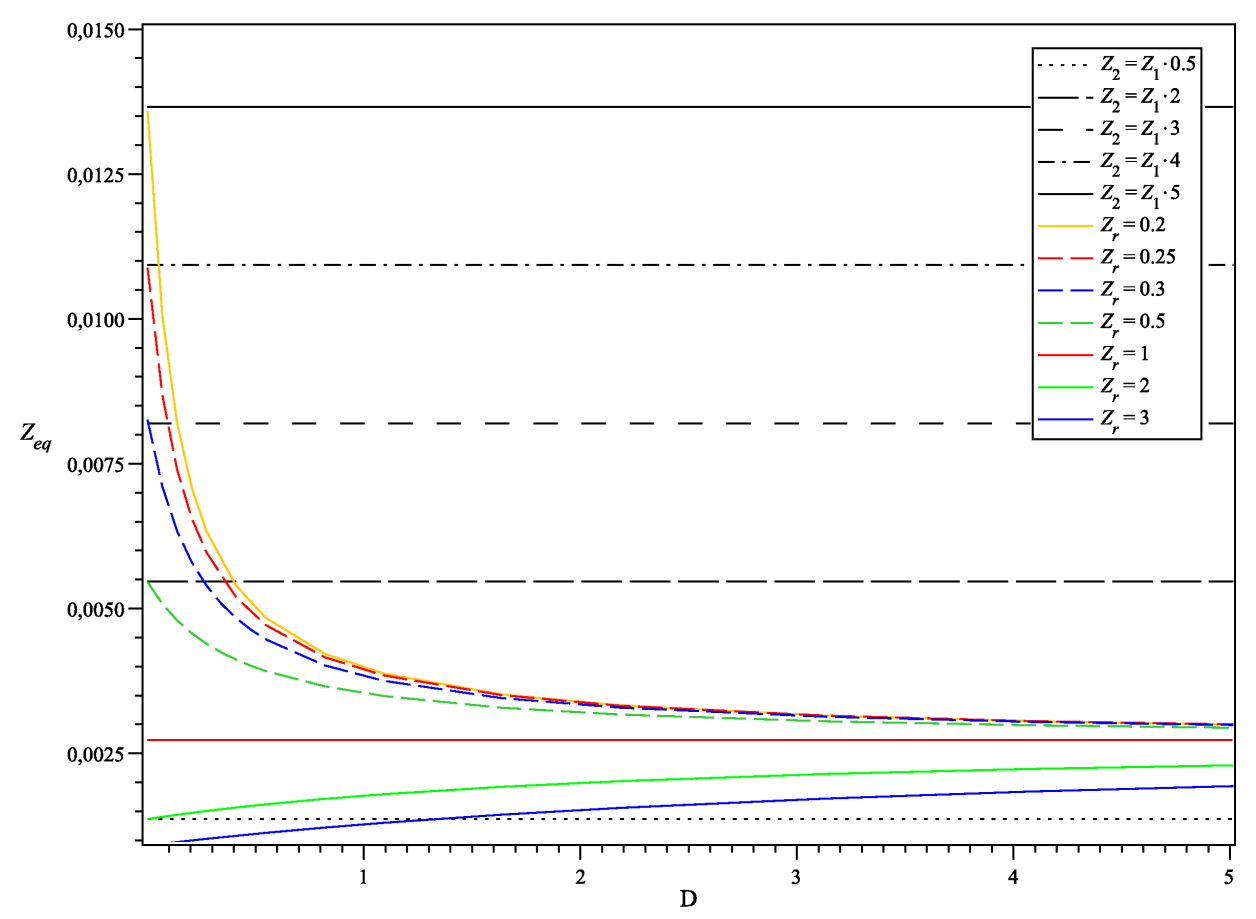

Figure 10. Equivalent figure of merit $v s$. the number of pairs ratio with different figure of merit ratios.

Our results show that if the thermoelectric properties of both modules have the same value, $Z_{r}=1$, the equivalent figure of merit $Z_{e q}$ becomes independent of the number of pairs. On the other hand, if $Z_{r} \neq 1$, the equivalent figure of merit approaches $Z_{r}=1$, as the number of pairs increases. Meanwhile, 
if the number of pairs ratio decreases, the value of $Z_{e q}$ tends to the figure of merit that satisfies the $Z_{r}$. Notice that the value of $Z_{e q}$ is not greater that the values of the figure of merit for each TEM of the system, independently of the number, $n$ or $m$, of the number of pairs. Thus, the result is according to the theorem of Bergman [17], which says that the equivalent figure of merit of the TEM, can only be lower than the highest $Z$ of the more efficient TEM. The value of $Z_{e q}$ is affected by the figure of merit ratio $Z_{r}$. When $Z_{r}$ is lower than the unit, or $Z_{2}>Z_{1}$, it is possible to increase the values of the equivalent figure of merit by having a lower number of pairs ratio $n>m$, but it is not possible to get a higher value than the $Z_{2}$ that satisfies the $Z_{r}$. Our analysis is consistent with the obtained results for thermoelectric generator systems [8] because of the generality of Bergman Theorem.

\subsection{Simplified Approaches: Previously Considered Cases}

Finally, in this section, we derive some results previously obtained for more simple models.

From Equation (18), by setting $q_{c}=0$, it is possible to get the full solution for the temperature ratio $\theta$,

$$
\theta=\frac{1}{\varphi(1-\varphi \rho)-\frac{1}{2} \varphi^{2} \frac{1-\varphi \rho+2 \rho}{Z_{e q} T_{c}}+1}
$$

In order to proceed with the approaches, it is necessary to define a new temperature ratio $\theta_{h}$ :

$$
\theta_{h}=\frac{1}{\theta}=\varphi(1-\varphi \rho)-\frac{1}{2} \varphi^{2} \frac{1-\varphi \rho+2 \rho}{Z_{e q} T_{c}}+1 .
$$

Now, solving $\frac{d \theta_{h}}{d \varphi}=0, \varphi_{\max }$ can be approximated through a power series expansion, leading to:

$$
\varphi_{\max }=Z_{e q} T_{c}-\left(\frac{1}{2} Z^{2} T_{c}^{2}+2 Z_{e q} T_{c}\right) \rho+O\left(\rho^{2}\right)
$$

and finally, substituting $\varphi_{\max }$ into Equation (34) yields:

$$
\theta_{h(\max )}=\varphi_{\max }\left(1-\varphi_{\max } \rho\right)-\frac{1}{2} \varphi_{\max }^{2} \frac{\left(1-\varphi_{\max } \rho+2 \rho\right)}{Z_{e q} T_{c}}+1
$$

In the limit $\rho \rightarrow 0$ or when $K_{c}>\left(m K_{1}+n K_{2}\right)$, the maximum dimensionless current and temperature ratio are respectively reduced to:

$$
\begin{aligned}
\varphi_{\max } & =Z_{e q} T_{c} \\
\theta_{h(\max )} & =\frac{1}{2} Z_{e q} T_{c}+1
\end{aligned}
$$

We highlight the fact that if the thermoelectric properties of the two modules are the same, i.e., $\alpha_{1}=\alpha_{2}=\alpha, R_{1}=R_{2}=R$ and $K_{1}=K_{2}=K$, then the equivalent figure of merit becomes the ordinary one, and from Equations (37) and (38) we obtain,

$$
\begin{aligned}
\varphi_{\max } & =Z T_{c} \\
\theta_{h(\max )} & =\frac{1}{2} Z T_{c}+1
\end{aligned}
$$

Equations (39) and (40) have been previously obtained by many authors [5,6]. 


\section{Conclusions}

Using our proposed approach to analyze a composite TEC system, formed by two TEMs, with different thermoelectric properties each, connected thermally in parallel and electrically in series, equivalent thermoelectric properties have been derived. These equivalent properties depend on all thermoelectric parameters of the composite TEC system, namely Seebeck coefficients, $\alpha_{(1,2)}$, thermal conductivity, $K_{(1,2)}$, the electrical resistance, $R_{(1,2)}$, of each TEM and external thermal conductances $K_{(c, h)}$. The corresponding dimensionless heat balance equations may be very useful for the design of composite thermoelectric systems, because they show the relation of the thermoelectric parameters of the TEM components of the system. The main parameters of the TEC system are external thermal conductances of heat exchangers, $K_{c h}$, and the figures of merit ratio, $Z_{r}$. The obtained results from this approach show the effect of two or more thermoelectric parameters on the $C O P$ and $q_{c}$ of the system. In this work, it is shown that the maximum values for $C O P$ and $q_{c}$ are determined by the external thermal conductances ratio $K_{c h}$, with the condition $K_{c}<K_{h}$. In general, we have shown (see Section 4) that our approach permits us to determine the optimal values of $q_{c}$ and $C O P$ for different working conditions determined. The obtained results in this work are useful for designing composite TEC systems with thermal coupling, $K_{c h}$. We show the consistency of our approach obtaining the COP as a function of the input voltage for a commercial thermoelectric module connected electrically in series. Furthermore, we have derived results previously obtained by many authors as limit cases of our approach. Finally, we point out that the proposed approach in this work can be easily extended to include many TEMs with different thermoelectric properties, each one connected thermally in parallel and electrically in series.

\section{Acknowledgments}

M.A.O.R. This work was financially supported by research grant 20150488 of Instituto Politecnico Nacional, Mexico. C.Y.F.N. was partially financially supported by CONACyT, Mexico (grant no. 514381). The authors acknowledge the editorial assistance of the editors to improve the manuscript.

\section{Author Contributions}

M.A.O.R. designed research; C.Y.F.N. calculated the data; M.A.O.R. and C.Y.F.N. analyzed data; I.L. supervised the dimensionless analysis, read and commented on the manuscript; M.A.O.R. and C.Y.F.N. wrote the paper. All authors have read and approved the final manuscript.

\section{A. Effect of the Thermoelectric Parameters in COP and $Q_{c}$}

In this Appendix, we list figures showing the effect of the thermoelectric parameters on $C O P$ and $q_{c}$ of the system. Notice that several results are included in the 3D figures discussed in the above sections. The figures in this Appendix show important limit intervals of the thermoelectric parameters. 


\section{A.1. Cooling Capacity $q_{c}$}

We reproduce several results for $q_{c}$ in terms of thermal conductances, $K_{c}$ and $K_{h}$, electrical current, $\varphi$, and the figure of merit, $Z$.

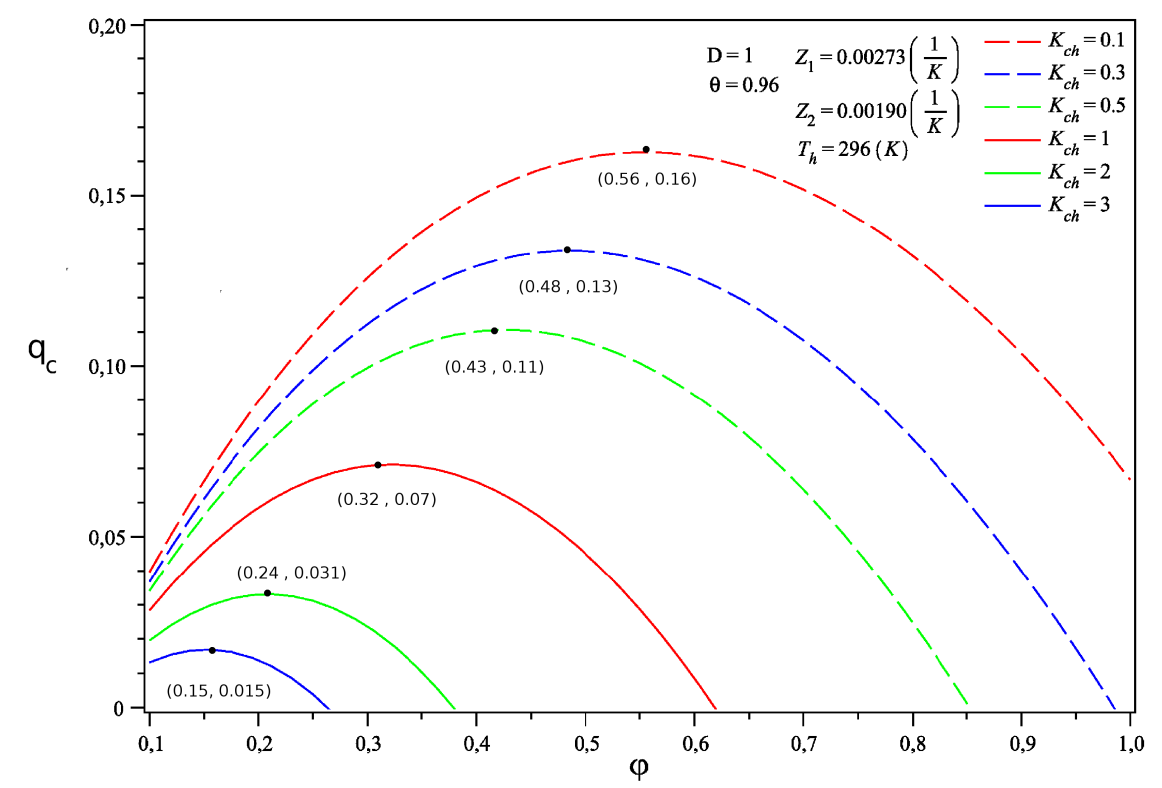

Figure 11. Cooling capacity vs. dimensionless current with different external thermal conductances ratios.

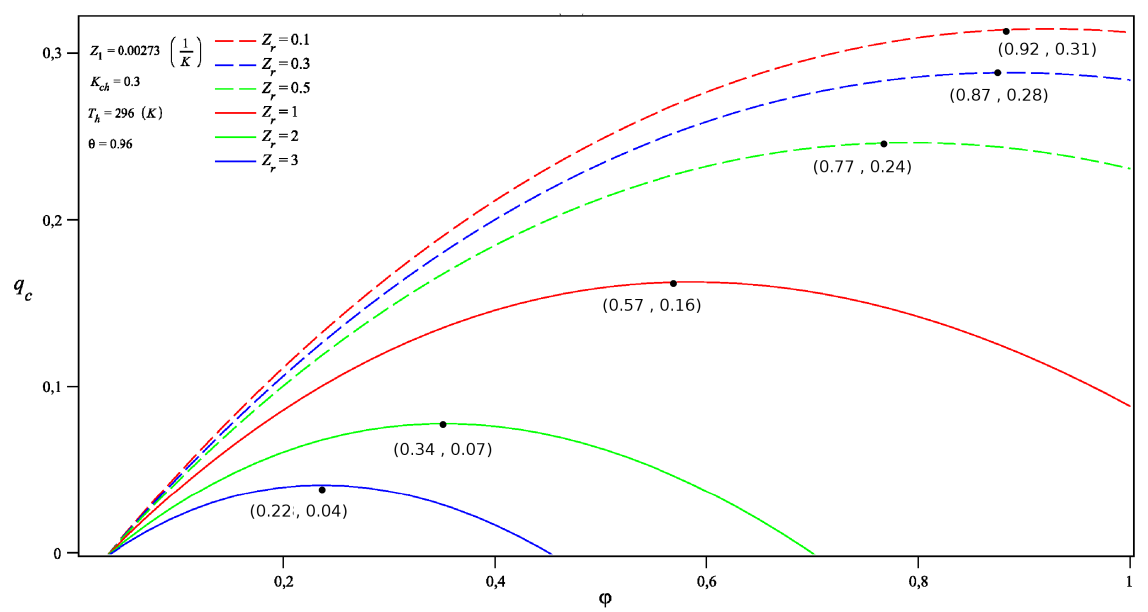

Figure 12. Cooling capacity vs. dimensionless current with different figure of merit ratios.

Figure 11 shows the effect of the external conductances, $K_{c h}=K_{c} / K_{h}$, on the cooling capacity $q_{c}$. At the same electrical current $\varphi$, we can obtain different maximum values for $q_{c}$ for a given value of $Z_{r}=Z_{1} / Z_{2}$.

On the other hand, for a given $K_{c h}$, the behavior of cooling capacity $q_{c} v s$. the electrical current is shown in Figure 12 when we change the figures of merit ratio $Z_{r}$. 


\section{A.2. Coefficient of Performance}

In this Appendix section, we obtain several results for $C O P$ in terms of thermal conductance, $K_{c h}$, electrical current, $\varphi$, and figure of merit, $Z$, see Figures 13-15. Our results contained in the Appendix show important intervals of limit values for the thermoelectric parameters. For example, as $Z_{r}$ increases in the TEMs, clearly, for the same current, we have different cooling capacities. In Figure 12, we show that there are intervals for $\varphi$ in which $q_{c}$ is zero for a given $Z_{r}$ value. If $Z$ of any TEM increases, $q_{c}$ increases also. Figure 4 shows some intervals for maximum values of $q_{c}$ for this condition.

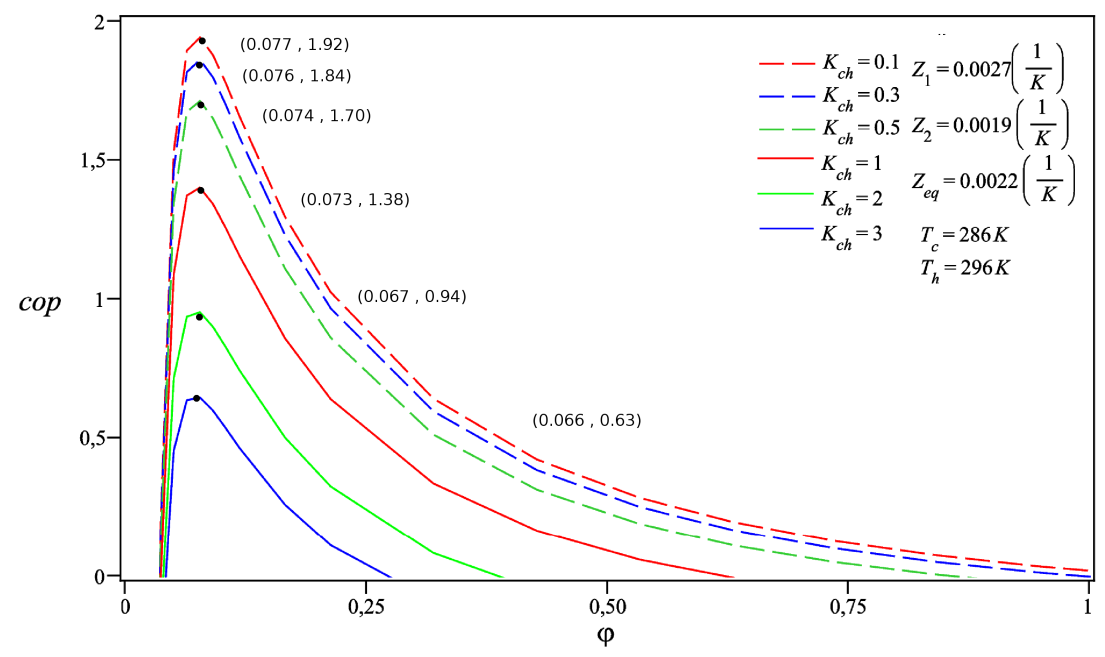

Figure 13. COP vs. dimensionless current with different external conductances ratios.

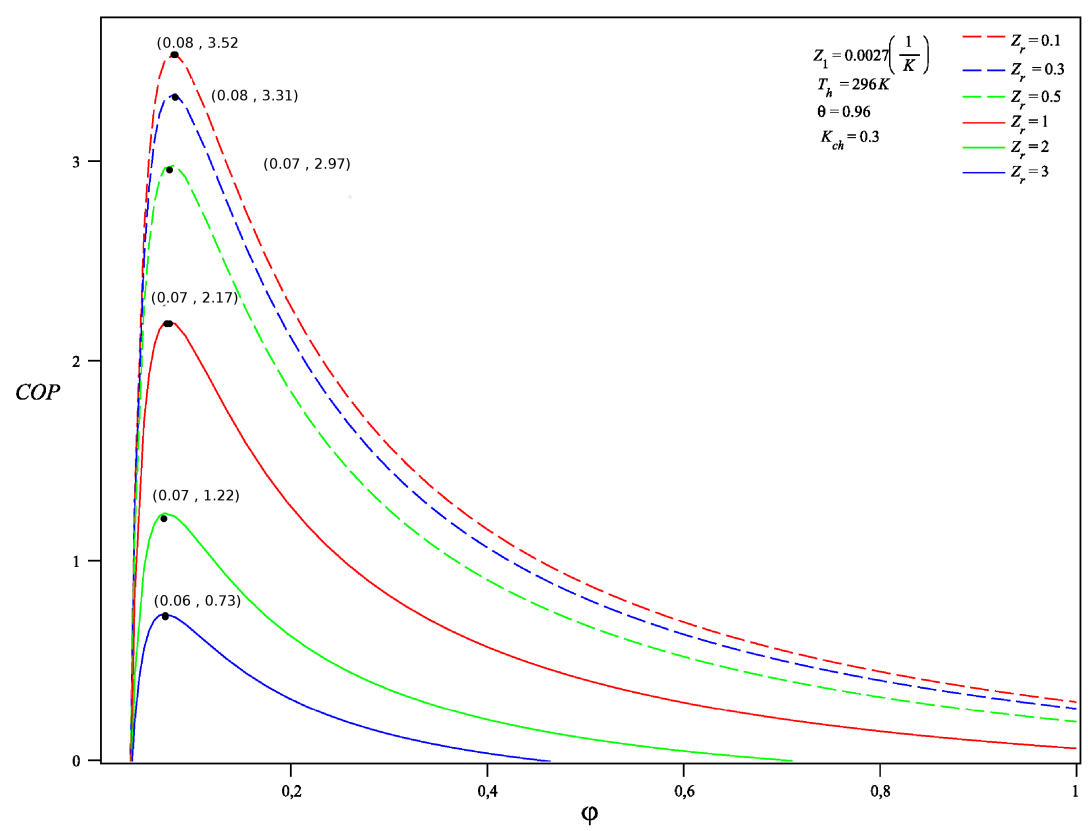

Figure 14. COP vs. dimensionless current with different figure of merit ratios. 


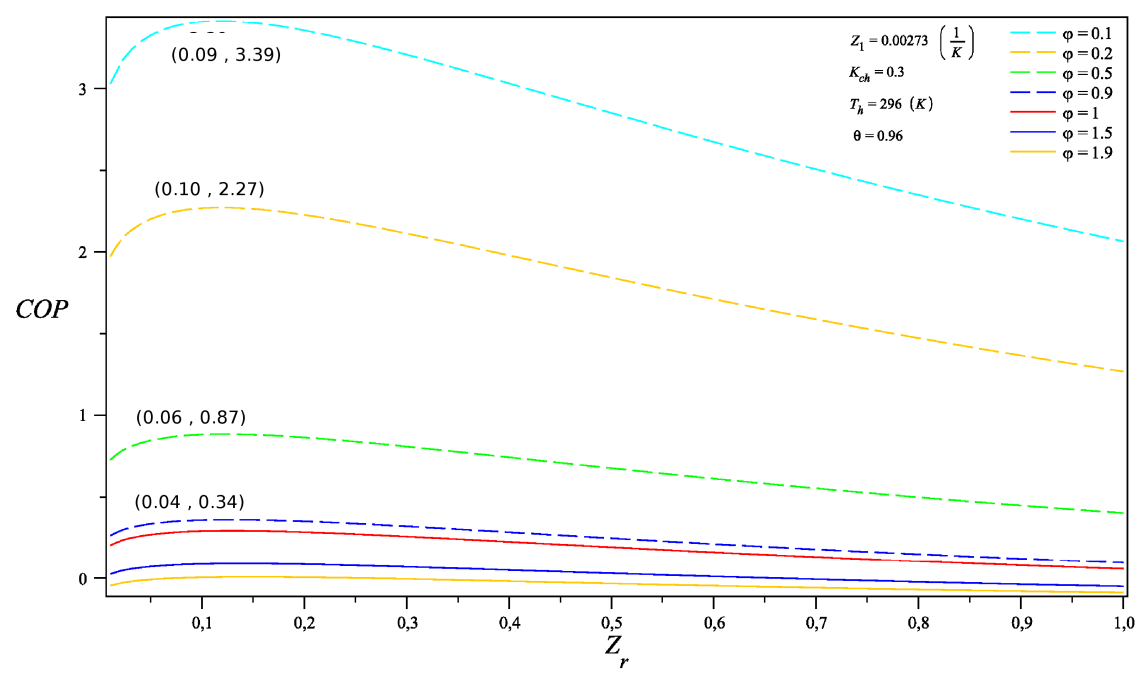

Figure 15. COP $v s$. the figure of merit ratio with different dimensionless currents.

\section{Conflict of Interest}

The authors declare no conflicts of interest.

\section{Nomenclature}

$\begin{array}{cll}C O P & \text { Coefficient of performance } & \\ D & \text { Number of pair ratio } & (A) \\ I & \text { Electric current through thermoelements } & \\ K & \text { Thermal conductance of thermoelement } & \\ K_{c} & \text { Thermal conductance of cold-end heat exchanger } & \left(\frac{W}{K}\right) \\ K_{c h} & \text { Thermal conductance of heat exchangers ratio } & \\ K_{h} & \text { Thermal conductance of hot-end heat exchanger } & \left(\frac{W}{K}\right) \\ m & \text { Total number of thermoelements at first TEM } & \\ n & \text { Total number of thermoelements at second TEM } & \\ q_{c} & \text { Dimensionless cooling capacity } & \\ q_{h} & \text { Dimensionless heat rejection } & \\ Q_{c} & \text { Cooling capacity of the TEC system } & (W) \\ Q_{h} & \text { Heat rejection of the TEC system } & (W) \\ R & \text { Electric resistance of thermoelement } & (\Omega) \\ T_{1} & \text { Hot end temperature of TEMs } & (K) \\ T_{2} & \text { Cold end temperature of TEMs } & (K) \\ T_{c} & \text { Temperature of the cold reservoir } & (K) \\ T_{h} & \text { Temperature of the hot reservoir } & (K) \\ V & \text { Input voltage } & (V) \\ Z & \text { Figure of merit } & \left(\frac{1}{K}\right) \\ Z_{r} & \text { Figures of merit ratio } & \\ Z_{e q} & \text { Equivalent figure of merit } & \end{array}$


Greek letters

$\begin{array}{cll}\alpha & \text { Seebeck coefficient of thermoelement } & \left(\frac{V}{K}\right) \\ \delta & \text { Cold conductance ratio } & \\ \Delta T & \text { Temperature across the TEMs } & (K) \\ \rho & \text { Hot conductance ratio } & \\ \theta & \text { Temperature of heat reservoirs ratio } & \\ \varphi & \text { Dimensionless current } & \\ \mu & \text { Coupling factor }\end{array}$

Subscripts

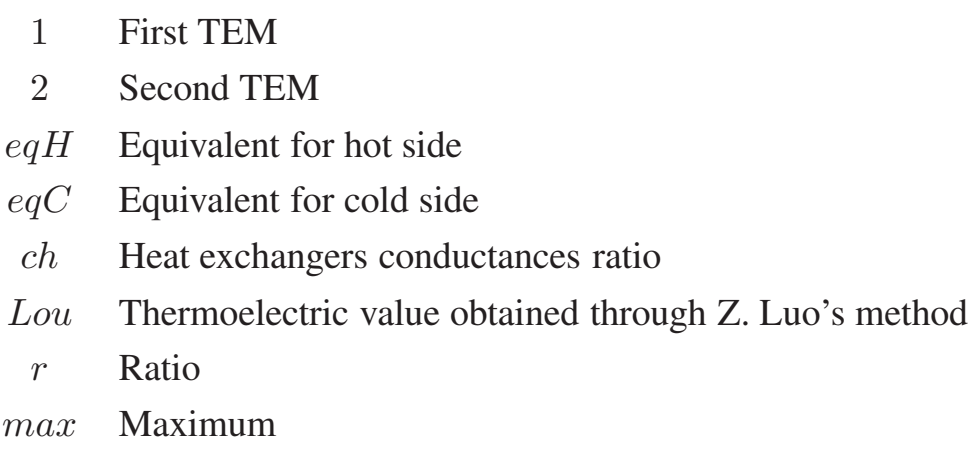

\section{References}

1. Rowe, D.M. Thermoelectric Handbook, Macro to Nano; CRC Press: Taylor and Francis Group, Boca Raton, FL, USA, 2006.

2. Nolas, G.S.; Sharp, J.; Goldsmid, H.J. Thermoelectrics: Basic Principles and New Materials Developments; Springer Verlag: Berlin, Germany, 2001.

3. Shakouri, A. Recent Development in Semiconductor Thermoelectric Physics and Materials. Ann. Rev. Mater. Res. 2011, 41, 399-431.

4. Riffat, S.B.; Ma, X. Thermoelectrics: A Review of Present and Potential Applications. Appl. Therm. Eng. 2003, 23, 913-935.

5. Yamanashi, M. A New Approach to Optimum Design in Thermoelectric Cooling Systems. J. Appl. Phys. 1996, 80, 5494-5502.

6. Xuan, X.C. Investigation of Thermal Contact Effect on Thermoelectric Coolers. Energ. Convers. Manage. 2003, 44, 399-410.

7. Pearson, M.R.; Lents, C.E. Dimensionless Optimization of Thermoelectric Cooler Performance when Integrated within a Thermal Resistance Network. In Proceedings of the ASME 2013, International Mechanical Engineering Congress and Exposition, San Diego, CA, USA, 15-21 November 2013; pp. V010T11A087-V010T11A087.

8. Apertet, Y.; Ouerdane, H.; Goupil, C.; Lecoeur, P. Thermoelectric Internal Loops inside Inhomogeneous System. Phys. Rev. B 2012, 85, 033201.

9. Apertet, Y.; Ouerdane, H.; Glavatskaya, O.; Goupil, C.; Lecoeur, P. Optimal Working Conditions for Thermoelectric Gnerators with Realistic Thermal Coupling. Europhys. Lett. 2012, 97, 28001. 
10. Vargas-Almeida, A.; Olivares-Robles, M.A.; Camacho-Medina, P. Thermoelectric System in Different Thermal and Electrical Configurations: Its Impact in the Figure of Merit. Entropy 2013, 15, 2162-2180.

11. Camacho-Medina, P.; Olivares-Robles, M.A.; Vargas-Almeida, A. Maximum Power of Thermally and Electrically Coupled Thermoelectric Generators. Entropy 2014, 16, 2890-2903.

12. Ioffe, A. Semiconductor Thermoelements and Thermoelectric Cooling; Infosearch: London, UK, 1957.

13. Home page of TE Technology. Available online: http://tetech.com (accessed on 1 February 2015).

14. Home page of Thermal Force. Available online: http://thermalforce.de (accessed on 1 June 2015).

15. Luo, Z. A Simple Method to Estimate the Physical Characteristics of a Thermoelectric Cooler from Vendo Datasheet. Electon. Cool. 2008, 14, 22-27.

16. Series-Parallel Modules. Available online: https://tetech.com/Peltier-thermoelectric-cooler-modules/series-p (accessed on 5 June 2015)

17. Bergman, D.J.; Levy, O. Thermoelectric Properties of a Composite Medium. J. Appl. Phys. 1991, 70, 6821-6833.

(C) 2015 by the authors; licensee MDPI, Basel, Switzerland. This article is an open access article distributed under the terms and conditions of the Creative Commons Attribution license (http://creativecommons.org/licenses/by/4.0/). 